\title{
Dyeing mechanism and optimization of polyamide 6,6 functionalized with double barrier discharge (DBD) plasma in air
}

\author{
Fernando Ribeiro Oliveira ${ }^{\mathrm{a}, 1}$, Andrea Zille $^{\mathrm{b}, *, 1}$, Antonio Pedro Souto $^{\mathrm{b}}$ \\ a Departamento de Engenharia Têxtil, Universidade Federal do Rio Grande do Norte UFRN, 59.072-970 Natal, Brazil \\ b 2C2T - Centro de Ciência e Tecnologia Têxtil, Departamento de Engenharia Têxtil, Universidade do Minho, 4800-058 Guimarães, Portugal
}

\section{A R T I C L E I N F O}

\section{Article history:}

Received 15 November 2013

Received in revised form

17 December 2013

Accepted 20 December 2013

Available online 30 December 2013

\section{Keywords:}

Dielectric barrier discharge (DBD)

Dyeing mechanism

Direct dye

Surface characterization

Plasma

Polyamide

Nylon

XPS

\begin{abstract}
A B S T R A C T
The physico-chemical improvements occasioned by DBD plasma discharge in dyeing process of polyamide 6,6 (PA66) fibers were investigated. The SEM, fluorescence microscopy, UV-vis spectroscopy, surface energy, FTIR, XPS and pH of aqueous extracts confirm the high polar functionalization of PA66 fibers due to plasma incorporation of oxygen atoms from atmospheric air. DBD plasma-generated reactive species preferentially break the $\mathrm{C}-\mathrm{N}$ bonds, and not the aliphatic $\mathrm{C}-\mathrm{C}$ chain of PA66. Formation of low-molecular weight acidic molecules that act as dye "carrier" and creation of micro-channels onto PA66 surface seems to favor dye diffusion into the fiber cores. Plasma treatment allows high level of direct dye diffusion and fixation in PA66 fibers at lower temperatures and shorter dyeing times than traditional dyeing methods.
\end{abstract}

(C) 2013 Elsevier B.V. All rights reserved.

\section{Introduction}

In recent years, textile industry in developed countries is challenging an increasing global competition due to the changed world market conditions. This aspect forced western countries to exploit their high technical skills in the development of textile materials for high quality and technical performances [1]. Moreover, the increasing environmental and health concerns owing to the large quantities of water and hazardous chemicals used in the conventional textile finishing techniques lead to the development of new technologies. In recent decades, plasma technology has assumed a great importance among all available surface modification processes. It is a dry, environmental- and worker-friendly method to achieve surface alteration without modifying the bulk properties of the materials [2]. In particular, non-thermal plasmas are especially suited because most textile materials are heat sensitive polymers $[3,4]$. Among plasma technologies, atmospheric plasma is an alternative and cost-competitive method to wet chemical treatments,

\footnotetext{
* Corresponding author at: 2C2T - Centro de Ciência e Tecnologia Têxtil, Departamento de Engenharia Têxtil, Universidade do Minho, Campus de Azurém, 4800-058 Guimarães, Portugal. Tel.: +351 226074900; fax: +351 226099157.

E-mail address: azille@2c2t.uminho.pt (A. Zille).

1 These authors contributed equally to this work.
}

thus avoiding the need of expensive vacuum equipment and allowing continuous and uniform processing of fibers surfaces [5]. The dielectric barrier discharge technology (DBD) in air is one of the most effective non-thermal atmospheric plasma sources and has been attracting increasing interest for industrial textile applications [6-8].

In general, the conventional dyeing processes have a low yield, and the percentage of dye lost in the effluents can reach up to $50 \%$. Besides the obvious esthetic problem, dye wastewaters without an appropriate treatment can persist in the environment for extensive periods of time and are deleterious not only for the photosynthetic processes of the aquatic plants but also for all the living organisms since the degradation of these can lead to carcinogenic substances [9]. In this context, plasma technology has proved to intensify dyeing rates of textile polymers improving the diffusion of dye molecules into the fibers, to enhance color fastness and wash resistance of fabrics, to increase adhesion of coatings, and to modify the wettability of several fibers and fabrics such as cotton [10-13], polyamide [14-16], polyester [17,18], polypropylene $[19,20]$, silk [21] and wool [22-25]. The higher dye exhaustion provided by plasma application decreases the amount of dyestuff and water necessary for a desired shade. The possibility of reusing the water effluent contributes to a reduction of the effluent load with a significant diminution in costs and environmental impact [26]. 
The main objective of this study aims to investigate the effects and key parameters of DBD plasma discharge on polyamide 6,6 (PA66) fibers and to understand the nature and the mechanism of dye adsorption process promoted by DBD plasma. Previous investigations on the dyeing of polyamide fibers have studied various properties such as, adsorption, kinetics, diffusion, attachment and equilibrium [27]. However, an investigation proposing a dyeing mechanism, which can explain the physico-chemical improvements occasioned by DBD plasma discharge in the dyeing process, was never performed. Scanning electron microscope (SEM), static and dynamic contact angle measurements, X-ray photoelectron spectroscopy (XPS), Fourier transform infrared spectroscopy (FTIR), Fluorescence microscopy, UV-vis spectroscopy, surface energy, conductivity and $\mathrm{pH}$ of aqueous extraction have been used to study the changes of surface morphology, wettability and chemical composition of the plasma-treated polyamide fabrics.

\section{Materials and methods}

\subsection{Materials}

Commercial polyamide 6,6 (PA66) fabric with a warp density of 42 threads $\mathrm{cm}^{-1}$, a weft density of 30 threads $\mathrm{cm}^{-1}$ and a surface density of $95 \mathrm{~g} \mathrm{~m}^{-2}$ was used in this study. The samples were pre-washed with a $1 \mathrm{~g} \mathrm{~L}^{-1}$ of non-ionic detergent solution at $30^{\circ} \mathrm{C}$ for $30 \mathrm{~min}$ and then rinsed with water for another $15 \mathrm{~min}$, before dielectric barrier discharge (DBD) plasma treatment, in order to minimize contaminations. The direct dye (C.I. Direct Orange 57) was supplied by Dystar Textil farben, Frankfurt, Germany. All the other reagents were analytical grade purchased from Sigma-Aldrich, St. Louis, MO, USA and used without further purification.

\subsection{Plasma treatment}

The DBD plasma treatment was conducted in a semi-industrial prototype machine (Softal Electronics $\mathrm{GmbH} /$ University of Minho) working at room temperature and atmospheric pressure, using a system of metal electrode coated with ceramic and counter electrodes coated with silicon with $50 \mathrm{~cm}$ effective width, gap distance fixed at $3 \mathrm{~mm}$ and producing the discharge at high voltage $10 \mathrm{kV}$ and low frequency $40 \mathrm{kHz}$. The discharge power supplied by the electrodes and the speed may vary, with maximum discharge of $1.5 \mathrm{~kW}$ and speed of $60 \mathrm{~m} \mathrm{~min}^{-1}$. The machine was operated at the optimized parameters: $1 \mathrm{~kW}$ of power, velocity of $4 \mathrm{~m} \mathrm{~min}^{-1}, 5$ passages corresponding to a dosage of $2.5 \mathrm{~kW} \mathrm{~min}^{-2}$ as previously reported [15]. Plasmatic dosage was defined by the Eq. (1):

Dosage $=\frac{N \cdot P}{v \cdot l}$

where, $N$, number of passages; $P$, power $(\mathrm{W}) ; v$, velocity $\left(\mathrm{m} \mathrm{min}^{-1}\right)$; and $l$, width of treatment $(0.5 \mathrm{~m})$.

\subsection{Scanning electron microscopy (SEM)}

Morphological analyses of polyamide samples were carried out with an ultra-high resolution Field Emission Gun Scanning Electron Microscopy (FEG-SEM), NOVA 200 Nano SEM, FEI Company. Secondary electron images were performed with an acceleration voltage between 5 and $10 \mathrm{kV}$. Backscattering Electron Images were made with an acceleration voltage of $15 \mathrm{kV}$. Samples were covered with a film of Au-Pd (80-20 wt \%) in a high-resolution sputter coater, 208HR Cressington Company, coupled to with a MTM-20 Cressington High Resolution Thickness Controller.

\subsection{Contact angle measurement and surface energy}

The water wettability and surface energy of PA66 fabrics treated and untreated with plasma were characterized with Dataphysics equipment using OCA20 software with video system for the capturing of images in static and dynamic modes. The polar (YD) and dispersive (YP) components of the surface energy $(Y)$ were calculated by using the Wu method (harmonic-mean) as previously reported [15]. Since it is necessary at least two liquids for the calculation of surface energy, three liquids with known surface energy and surface energy components were used in this study, namely: distilled water (Y: 72.8; YD: 29.1; YP: 43.7), polyethylene glycol 200 (PEG) (Y: 43.5; YD: 29.9; YP: 13.6), and glycerol (Y: 63.4; YD: 37.4; YP: 26.0).

\subsection{Fourier transform infra-red (FTIR)}

The Attenuated Total Reflectance ATR-FTIR spectra of the polyamide samples (with and without plasma treatment) after and before a blank dye process were recorded in a Nicolet Avatar 360 FTIR spectrophotometer (Madison, USA). The spectra were collected in the region of $4000-400 \mathrm{~cm}^{-1}$ and at a resolution of $16 \mathrm{~cm}^{-1}$. For the samples obtained after aqueous extraction, the measurement of FTIR was carried out into potassium bromide $(\mathrm{KBr})$ pellets. $2 \mathrm{mg}$ of lyophilized aqueous extraction were grinded with $100 \mathrm{mg}$ dry $\mathrm{KBr}$, and pressed into a mold in a uniaxial hydraulic press under a load of $0.9 \mathrm{MPa}$ to obtain IR-transparent pellets. The infrared spectra were recorded with a FTIR system 2000 (Perkin Elmer, USA), in transmittance mode in the region of $4000-400 \mathrm{~cm}^{-1}$ and a resolution of $4 \mathrm{~cm}^{-1}$.

\subsection{X-Ray photoelectron spectroscopy (XPS)}

XPS measurements were performed on aVG Scientific ESCALAB 200 A equipment with PISCES software for data acquisition and analysis. For analysis, an achromatic $\mathrm{Al}(\mathrm{Ka}) \mathrm{X}$-ray source operating at $15 \mathrm{kV}(300 \mathrm{~W})$ was used, and the spectrometer, calibrated with reference to $\mathrm{Ag} 3 \mathrm{~d} 5 / 2(368.27 \mathrm{eV})$, was operated in CAE mode with $20 \mathrm{eV}$ pass energy. Data acquisition was performed with a pressure lower them 1E-6 Pa. Deconvolution into sub-peaks was performed by least-squares peak analysis software, XPSPEAK version 4.1, using the Gaussian/Lorenzian sum function and Shirley-type background subtraction (or linear consideration of the data).

\subsection{Dyeing method}

The dyeing method, with a dye concentration of $0.25,0.5$ and $0.75 \mathrm{~g} \mathrm{~L}^{-1}$ in distilled water without auxiliaries, was carried out in a laboratorial "Ibelus" machine equipped with infra-red heating with a liquor ratio of $1: 40$ and $\mathrm{pH}$ around 4.5 using stainless steel dye-pots with $200 \mathrm{~cm}^{3}$ of capacity each. The program was started at $20^{\circ} \mathrm{C}$ and raised at a rate of $2^{\circ} \mathrm{C} \mathrm{min}-1$ up to $98^{\circ} \mathrm{C}$, and kept at this temperature for $60 \mathrm{~min}$. The absorption measurements for the calculation of dyebath exhaustion process with direct dye were obtained in a Unicam UV/vis UV2 spectrophotometer.

\subsection{Conductivity and $p H$ of aqueous extract}

Untreated and plasma treated polyamide fabric samples with dosage of $2.5 \mathrm{~kW} \mathrm{~min} \mathrm{~m}^{-2}$ were immersed in distilled water (liquor ratio $1: 40$ ) and submitted to dyeing process $\left(98^{\circ} \mathrm{C}\right.$ during $60 \mathrm{~min}$ ). The $\mathrm{pH}$ and conductivity $(\mathrm{mV})$ were measured with a WTW $\mathrm{pH}$ meter 538, Weinheim, Germany. 

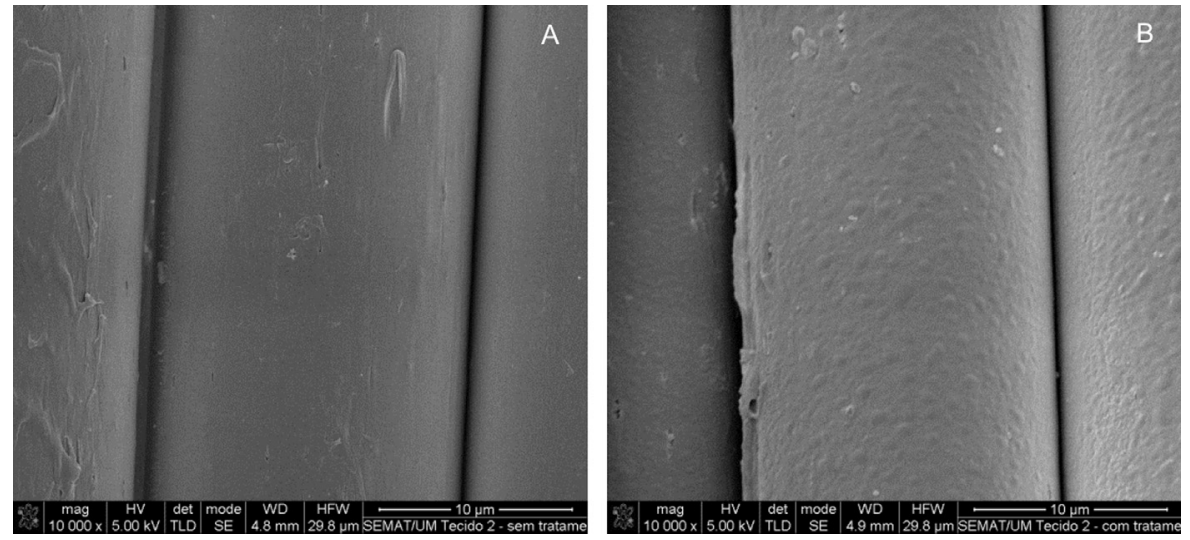

Fig. 1. SEM image of PA66 untreated (A) and plasma treated (B) fibers with dosage of $2.5 \mathrm{~kW} \mathrm{~min} \mathrm{~m}^{-2}$ with magnification of $10,000 \times$.

\subsection{Whiteness and color strength $(K / S)$}

The whiteness (Berger formulae) of the polyamide fabric after DBD treatment and color intensity of the dyed fabrics were measured by using a Datacolor Spectraflash SF 600 Plus CT spectrophotometer with D65 illuminant, over the range of 390-700 nm. The average of three reflectance measurements, taken at different positions on the dyed fabric, was adopted. The relative color strength (K/S values) was then established at standard illuminant D65 (LAV/Spec. Excl., d/8, D65/10 ${ }^{\circ}$ ), according to the Kubelka-Munk equation, where $\mathrm{K}$ and $\mathrm{S}$ stand for the light absorption and scattering, respectively. The shift of the coordinates of the color in the cylindrical color space, based on the theory that color is perceived by black-white ( $\mathrm{L}^{*}$, lightness), red-green $\left(\mathrm{a}^{*}\right)$, and yellow-blue $\left(b^{*}\right)$ sensations, was summarized by the overall color difference $\left(\Delta E^{*}\right)$ value. The value of $\Delta E^{*}$ represents the overall color difference between the sample and the standard.

\subsection{Washing, light and rubbing fastness}

The washing fastness was evaluated in accordance to the stipulated in standard ISO 105 C06, method A1S, at temperature of $40^{\circ} \mathrm{C}$. The rubbing fastness was evaluated according to standard ISO 105 X12:2001. The light fastness was performed in the Accelerated Weathering Tester (QUV) Spray LU-0819 from Q-PANEL, equipped with UVA 340 lamp, at $70^{\circ} \mathrm{C}$ and irradiation at $77 \mathrm{~W} / \mathrm{m}^{2}$ for light fastness test. The QUV test chamber simulated weather conditions. Exposure cycles of $2 \mathrm{~h}, 4 \mathrm{~h}, 8 \mathrm{~h}, 12 \mathrm{~h}, 24 \mathrm{~h}$ and $48 \mathrm{~h}$ were applied on samples until remarkable color degradation was observed.

\subsection{Fluorescence microscopy}

The level of dye penetration into fibers during the dye process was visualized by fluorescence microscopy on polyamide cross sections. The fibers were embedded into an epoxy resin and transversal cuts of the fibers with $15 \mathrm{~mm}$ were prepared using a microtome (Microtome Leitz). Fiber cross-sections were analyzed by a Fluorescence microscope LEICA DM 5000B at 40× magnification.

\section{Results and discussion}

\subsection{SEM morphological analysis}

To enhance the interaction between dyeing bath $(0.25,0.5$ or $0.75 \mathrm{~g} \mathrm{~L}^{-1}$ C.I. Direct Orange 57 in distilled water at $98^{\circ} \mathrm{C}$ adjusted to $\mathrm{pH} 4.5$, without auxiliaries and dyebath ratio $1: 40$ ) and hydrophobic PA66 fibers, the surface of the fabric was modified by DBD plasma. The optimal dosage was set-up to a value of

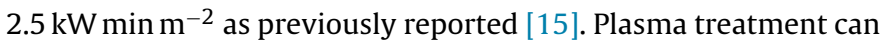
induce substantial morphological changes onto fiber surface, especially enhancing its roughness and consequently increasing surface energy, wettability and adhesion [28]. SEM images of untreated and DBD plasma treated PA66 fibers show that the topography of the fiber was uniformly altered after plasma treatment in the form of ripple-like structures of sub-micron size that were induced by plasma etching (Fig. 1). As previously stated, the highly reactive and energetic plasma species, achieved by DBD atmospheric pressure plasma treatment, promote synthetic fiber ablation (etching) and induce the increase of fiber surface roughness and hydrophilicitydependent properties [6,29]. Several mechanisms that illustrate the interaction between plasma and polymers via etching were suggested [30,31]. Besides the etching process, the energetic electrons generated on the material surfaces during plasmatic discharge can lead to scission of polymer molecular chains. This effect leads to the formation of free radicals that interact with other plasma generated reactive species, mainly related with oxygen and nitrogen molecules $\left(\mathrm{N}_{2}{ }^{+}, \mathrm{N}_{4}{ }^{+}, \mathrm{N}^{+}, \mathrm{O}_{2}{ }^{+}, \mathrm{H}_{2} \mathrm{O}^{+}, \mathrm{O}_{2}{ }^{-}, \mathrm{O}^{-}\right)$, producing new functional groups on the polymers surface [32]. Due to the free radicals actuation, the distinction between material removal and surface chemical modification becomes very difficult because these two processes occur simultaneously and synergistically, making the involved interaction mechanism (plasma/substrate) much more complex.

\subsection{Contact angle and surface energy measurements}

The surface properties of untreated and treated plasma polyamide fabrics were analyzed by static and dynamic contact angle measurements (Table 1). At the selected energy dosage, the static contact angle decreases from $133^{\circ}$ to $24^{\circ}$. Further plasma-energy dosages do not lead to significant differences in hydrophilicity. All plasma treated samples also show a dramatic dynamic contact angle reduction in less than $1 \mathrm{~s}$, as the untreated samples maintain hydrophobic contact angles. Using water, PEG and glycerol static contact angle values, the total surface energy $(\gamma)$, dispersive component $\left(\gamma_{\mathrm{D}}\right)$ and polar component $\left(\gamma_{\mathrm{P}}\right)$ of the polyamide fabric were calculated by $\mathrm{Wu}$ method [15]. As expected, total surface energy significantly increases after DBD plasma treatment. Initially, the dispersive and polar components of the polyamide fabric without treatment were $10.6 \mathrm{~mJ} \mathrm{~m}^{-2}$ and $1.3 \mathrm{~mJ} \mathrm{~m}^{-2}$, respectively. For a dosage of $2500 \mathrm{~W} \mathrm{~min} \mathrm{~m}^{-2}$, the dispersive component decreased until $7.0 \mathrm{~mJ} \mathrm{~m}^{-2}$ and the polar component has a huge increase up to $73.1 \mathrm{~mJ} \mathrm{~m}^{-2}$. Different physico-chemical factors can control the contact angle [33]. The intermolecular forces acting in the interface are partly a result of the London dispersive forces and their contribution to the surface 
Table 1

Static contact angles and surface energies of untreated and DBD plasma treated PA66 fabrics at days 1, 7 and 30.

\begin{tabular}{|c|c|c|c|c|c|c|}
\hline \multirow[t]{2}{*}{ Polyamide 6,6} & \multicolumn{3}{|c|}{ Contact angle $\left({ }^{\circ}\right)$} & \multicolumn{3}{|c|}{ Surface energy $\left(\mathrm{mJ} \mathrm{m}^{-2}\right)$} \\
\hline & $\theta_{\text {water }}$ & $\theta_{\mathrm{PEG}}$ & $\theta_{\text {glycerol }}$ & $\gamma$ & $\gamma_{\mathrm{D}}$ & $\gamma_{\mathrm{P}}$ \\
\hline Untreated & $132.6 \pm 3.8$ & $44.6 \pm 2.1$ & $131.7 \pm 3.3$ & 11.98 & 10.63 & 1.35 \\
\hline Plasma (day1) & $23.3 \pm 3.2$ & $22.9 \pm 2.4$ & $75.1 \pm 4.1$ & 80.19 & 7.04 & 73.15 \\
\hline Plasma (day7) & $42.3 \pm 3.7$ & $38.4 \pm 2.1$ & $84.0 \pm 4.6$ & 63.46 & 5.63 & 57.83 \\
\hline Plasma (day30) & $80.4 \pm 2.0$ & $44.4 \pm 1.5$ & $93.9 \pm 2.1$ & 26.90 & 14.01 & 12.89 \\
\hline
\end{tabular}

$\gamma_{\mathrm{D}}$, dispersive component; $\gamma_{\mathrm{P}}$, polar component.

energy is represented by $\gamma_{\mathrm{D}}$, while dipole-dipole interactions and hydrogen bond forces can be expressed through $\gamma_{\mathrm{P}}$ [34]. It is clear that the introduction of polar groups is responsible for the increase in wettability of the plasma modified PA66. Unfortunately, the surface does not show a stable behavior with time. It is well known that one of the major drawbacks of the plasma treatment with nonpolymerizing gases is the aging effect. After treatment, migration and reorientation of oxidized groups occur even at ambient temperature, leading in time to significant decreases in wettability and loss of surface oxidation [35]. The type of polymer, process, environmental and storage conditions were found to have significant effect on the aging process of plasma-treated substrate [36]. As expected, after 30 days of aging, plasma-treated PA66 samples showed a significant decrease in wettability (Table 1). However, the contact angles values of the plasma treated surfaces remain lower than the untreated sample. It is clear that plasma treated PA66 does not completely lose its wetting properties even after long aging periods. Moreover, a reasonable value suitable for dyeing at commercial scale is maintained until 7 days (Table 1 ). Improved plasma effect durability can be achieved with care in storage, relative humidity and temperature [37].

\subsection{FTIR analysis}

The ATR-FTIR spectrum of untreated PA66 sample shows the inherent bands of nylon at $3290 \mathrm{~cm}^{-1}$, which may be attributed to $\mathrm{N}-\mathrm{H}$ stretching vibrations (Fig. 2a). The peaks at 2930 and $2850 \mathrm{~cm}^{-1}$ may be attributed to the $\mathrm{CH}_{2}$ asymmetric and symmetric stretching vibrations, respectively [38]. The absorption band at $1630 \mathrm{~cm}^{-1}$, often referred to as amide I band, may be assigned to the amide carbonyl $\mathrm{C}=\mathrm{O}$ stretching vibrations. Instead, the amide II band at $1530 \mathrm{~cm}^{-1}$ may be attributed to $\mathrm{N}-\mathrm{H}$ bending motion. As expected, the presence of the hydrogen-bonded secondary amide is confirmed by the in-plane $\mathrm{N}-\mathrm{H}$ deformation vibration of the peak shoulder at $720 \mathrm{~cm}^{-1}$. The band at $680 \mathrm{~cm}^{-1}$ is attributed to the bending of $\mathrm{O}=\mathrm{C}-\mathrm{N}$ group [39]. After the blind dyeing, the plasma treated sample shows a significant increase in the intensity and broadening of the $\mathrm{C}=\mathrm{O}$ stretching band as well as of the bending band of the $\mathrm{O}=\mathrm{C}-\mathrm{N}$ group were observed. This may be an indication of the micro-environmental changes and oxygen addition on the fiber surface. On the other hand, the significant increase in intensities for the $\mathrm{N}-\mathrm{H}$ and asymmetric and symmetric $\mathrm{C}-\mathrm{H}$ stretching vibration bands at 3290, 2930 and $2850 \mathrm{~cm}^{-1}$, respectively, could also be assigned to the formation of low molecular weight etched material due to the air DBD treatment of the PA66 fabric [36]. According to the literature, the energetic electrons induced by plasma discharge using atmospheric air could cause the breaking of bonds with energy lower than $10 \mathrm{eV}$ in the outer layers of the PA66 polymer especially on the $\mathrm{C}-\mathrm{N}$ bonds, which is the weakest bond in the polymer chain [40]. These break chains, in contact with the reactive species created by plasma, can generate the formation of low-molecular weight molecules containing

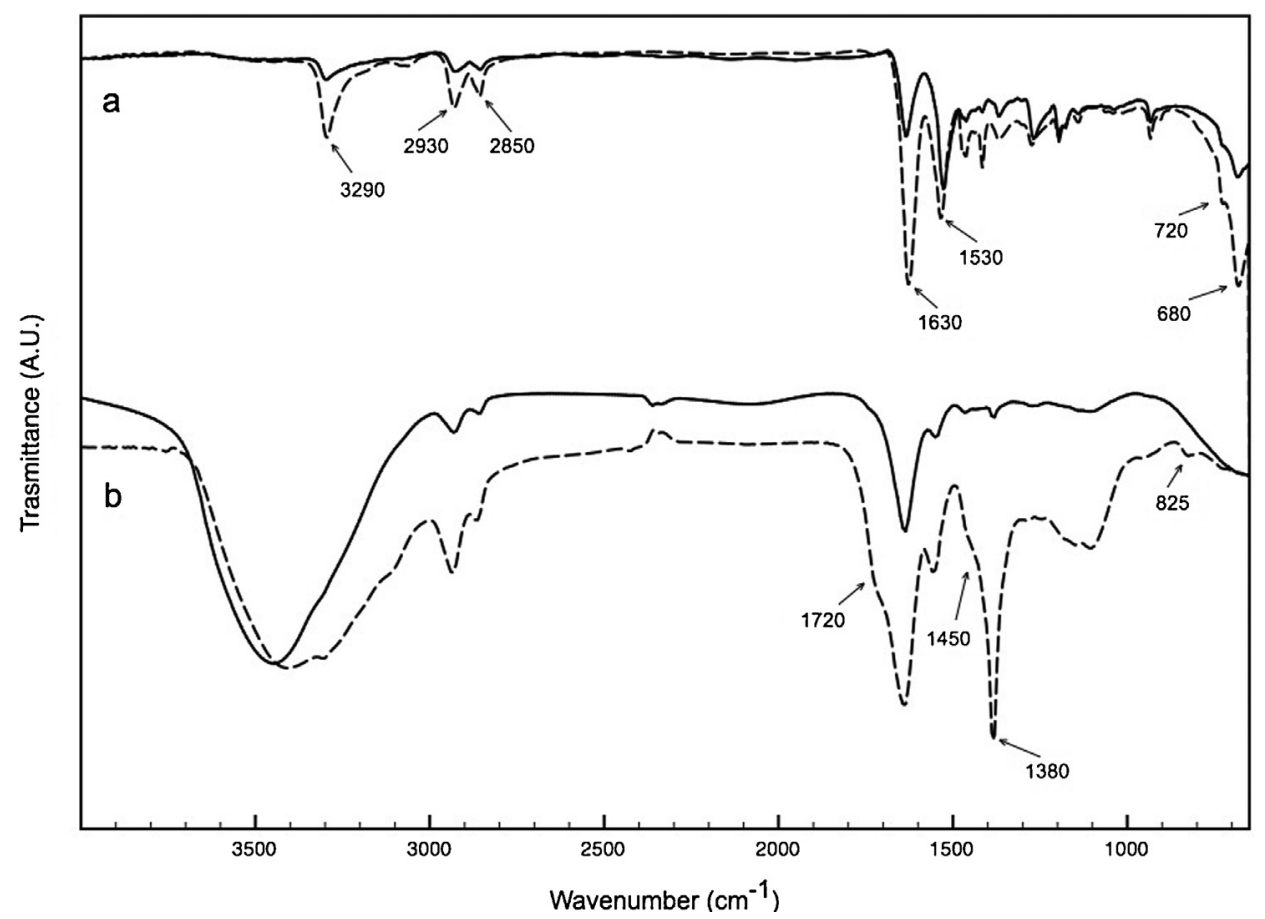

Fig. 2. (a) ATR-FTIR spectra of PA66 fabric and (b) and transmittance mode FTIR spectra of the aqueous extraction residue after blank dyeing process. With plasma treatment (dotted lines) and without plasma treatment (solid lines). 
Table 2

Chemical structure and relative chemical composition and atomic ratio determined by XPS of untreated and DBD plasma treated PA66 fabrics.

\begin{tabular}{lll}
\hline Polyamide 6,6 & $\begin{array}{l}{\left[-\mathrm{CO}-\mathrm{CH}_{2}-\left(\mathrm{CH}_{2}\right)_{2}-\mathrm{CH}_{2}-\mathrm{CO}-\mathrm{NH}-\mathrm{CH}_{2}-\right.} \\
\end{array}$ & $\left.\left(\mathrm{CH}_{2}\right)_{4}-\mathrm{CH}_{2}-\mathrm{NH}-\right]_{n}$ \\
\cline { 2 - 3 } & Untreated & Plasma treated \\
\hline Carbon (C) at [\%] & 74.3 & 60.5 \\
Oxygen (O) at [\%] & 16.3 & 28.5 \\
Nitrogen (N) at [\%] & 9.4 & 11.0 \\
Ratio O/C & 0.22 & 0.47 \\
Ratio N/C & 0.13 & 0.18 \\
\hline
\end{tabular}

carboxyl and amine groups, which are partially removed by aqueous extraction and could be identified in the effluent's residue. The transmission FTIR analysis of the aqueous extraction residues after a blank dyeing process (without dye) shows remarkable differences between untreated and plasma treated PA66 fabrics (Fig. 2b). The new shoulder peak at $1720 \mathrm{~cm}^{-1}$ adjacent to the increased amide $\mathrm{C}=\mathrm{O}$ band at $1630 \mathrm{~cm}^{-1}$ is a result of the additional contribution from carbonyl groups formed by air DBD treatment. The two characteristic IR peaks for $-\mathrm{CH}_{3}$ (intense peak at $1380 \mathrm{~cm}^{-1}$ ) and for $-\mathrm{CH}_{2}$ (a shoulder peak at $1450 \mathrm{~cm}^{-1}$ ) suggest that the hydrocarbon fragments from plasma treated PA66 are released in the aqueous medium [41]. However, the intense peak at $1380 \mathrm{~cm}^{-1}$ as well as the broad peaks between 1000 and $1200 \mathrm{~cm}^{-1}$ may be also attributed to the $\mathrm{C}-\mathrm{O}$ stretching of free and condensed $\mathrm{C}-\mathrm{OH}$ groups [42]. This last hypothesis is supported by the significant differences in conductivity and $\mathrm{pH}$ of the aqueous extracts of the PA66 before and after plasma treatment. Conductivity values increase from 57 to $211 \mathrm{mV}$ and $\mathrm{pH}$ values decrease from 5.5 to 3.1 confirming the presence of a significant concentration of acidic species on the effluent. According to Mattos et al., 2002, the appearance of a peak at $825 \mathrm{~cm}^{-1}$ can be related to the $\mathrm{NO}_{3}{ }^{-}$group [43].

\subsection{XPS analysis}

XPS analyses were performed to obtain a deeper understanding of the degree of chemical modification on the PA66 fibers surface. The increase of atomic ratio $\mathrm{O} / \mathrm{C}$ after DBD plasma treatment indicates a substantial incorporation of oxygen atoms onto the fabric surface (Table 2). The ratio N/C shows only a slight increase as previously observed [29]. Plasma etching may provoke chain scission in groups $\mathrm{C}-\mathrm{H}, \mathrm{C}-\mathrm{O}, \mathrm{C}-\mathrm{N}, \mathrm{C}-\mathrm{C}, \mathrm{N}-\mathrm{H}$ present in the PA66 fiber promoting the formation of reactive species such as $\mathrm{O}^{-}, \mathrm{N}, \mathrm{N}^{+}, \mathrm{O}, \mathrm{OH}^{-}$, $\mathrm{O}_{3}$, thus causing the decrease of carbon content and the increase of nitrogen and oxygen atoms $[36,44,45]$. However, due to the low energies involved in this specific treatment, the difference in nitrogen content could be arise from a surface cleaning effect that allows the fingerprint of the material bulk to appear on the spectra [46,47]. It is clear that the plasma treatment is able to increase the concentration of polar groups near the surface of the fabric mainly by the incorporation of oxygen atoms from atmospheric air, which significantly increases wettability $[46,48]$.

Deconvolution of the C1s core level of the untreated PA66 does not show significant deviation from the expected stoichiometric ratios of the individual carbon components (Fig. 3a). The peak at $285 \mathrm{eV}$ is attributed to aliphatic carbon atoms of the nylon structure $\left(-\mathrm{CH}_{2}-\mathrm{CH}_{2}-\right)$. The peak at $286 \mathrm{eV}$ represents the carbon atoms neighboring the amide nitrogen $\left(-\mathrm{NH}-\mathrm{CH}_{2}-\right)$ and that of at $287.8 \mathrm{eV}$ is assigned to the amide carbonyl group $(\mathrm{O}=\mathrm{C}-\mathrm{NH}-)$ [49]. These last two peaks have the same area as expected [50]. After plasma treatment a new peak attributed to the carboxyl acid group at $288.8 \mathrm{eV}$ (9.1\%) was observed [51]. A significant increase in $\mathrm{O}=\mathrm{C}-\mathrm{NH}-$ at $287.8 \mathrm{eV}$ (from 15.2 to $18.8 \%$ ) was also noticed due to the incorporation of oxygen atoms from atmospheric air
[7]. These results indicate that more polar functional groups are incorporated to PA66 fibers surface [15,52]. The peak assigned to the carbon atoms neighboring the amide nitrogen at $286 \mathrm{eV}$ does not show significant deviation owing to the fact that the primary amino groups $\mathrm{C}-\mathrm{NH}_{2}$ peak eventually generated by the chain scissions at the $\mathrm{C}-\mathrm{N}$ bond (as suggested previously in the FTIR analysis) have the same energy binding range of the $\mathrm{C}-\mathrm{NH}$. On the other hand, the slight growth (from 15.2 to $15.7 \%$ ) present in this peak could indicate that not all the carbons in the nylon surface can be converted into doubly bonded to oxygen by dielectric barrier discharge (DBD) processing in air [29]. The peak associated to the aliphatic carbons shows a remarkable decrease (from 69.6 to $56.4 \%$ ). These data, in addition to the FTIR analysis, suggest that direct aliphatic chain scission $\mathrm{C}-\mathrm{C}$ is not favored in the DBD processing of polymer surfaces $[7,53]$ and that the preferred oxidation sites are the methylene carbons immediately adjacent to the amide carbonyls [40]. Therefore, it is clear that the formation of low molecular weight molecules can occur, and through oxidation, this tends to form acidic and partially soluble species as previously confirmed by FTIR, conductivity and $\mathrm{pH}$ analysis of the aqueous extracts.

The deconvoluted $01 \mathrm{~s}$ spectrum of the untreated sample consists of two peaks (Fig. 3c). Theoretically, the main peak at $531.4 \mathrm{eV}$ attributed to the amide carbonyl oxygen should be the only oxygen specie present on the untreated substrate. However, due to the presence of low-level photo-oxidized central carbons on the PA66 surface (usual for commercial polymers), a second peak at $533.2 \mathrm{eV}$, corresponding to oxygen atoms singly bonded to carbon atoms, was observed $[3,44,50]$.

The deconvoluted 01s spectra of the plasma treated PA66 sample (Fig. 3d) also shows two peaks, however, the peaks previously attributed to the single bonded oxygen show a shift in binding energy (from $533.2 \mathrm{eV}$ to $532.7 \mathrm{eV}$ ) related to the presence of the carboxyl acid group [54]. These results suggest that the oxidation of the surface takes place, in an initial phase, mainly by the formation of $\mathrm{C}-\mathrm{O}$ bond that is dominant in the earliest stages of DBD treatment as previously stated $[7,46]$.

The N1s core level spectra of the untreated and plasma treated PA66 shows (Fig. 3e-f), as expected, a single and broad peak at $399.7 \mathrm{eV}$ imputed to the nitrogen of the $\mathrm{O}=\mathrm{C}-\mathrm{N}$ bond [55].

\subsection{Dyeing optimization}

Acid dyes are the most common in use for polyamide fibers. However, their use is restricted to those containing only one anionic group because of the lower concentration of cationic ammonium groups on the PA surface ( $10 \%$ that of wool). Moreover, acid dyes exhibit some drawbacks such as poor leveling and wet fastness properties and sometimes an excessively low dyebath $\mathrm{pH}$. Direct dyes can be applied on nylon in a very similar manner to acid dyes, sharing some of their problems such as the sensitivity to nylon structural variations. However, direct dyes are economically more attractive to produce some full bright reds, scarlets, dark browns, greens and blacks. In this context, plasma pre-treatment allows the dyeing of nylon with direct dyes without auxiliaries and at lower temperature and dye concentration $[15,56]$. The exhaustion test shows that dyeing kinetic is greatly influenced by plasma treatment. Dye uptake was monitored measuring both $\mathrm{K} / \mathrm{S}$ values of the dyed fabrics and dyebath concentrations before and after plasma treatment. As observed in Fig. 4, K/S values obtained in plasma pretreated sample are significantly higher when compared with the untreated ones. Furthermore, after $60 \mathrm{~min}$, dyebath concentration is completely exhausted in the plasma treated sample; hence this never occurs in the untreated sample. An additional dyeing test in the plasma treated PA66 carried out at $80^{\circ} \mathrm{C}$ shows the same $\mathrm{K} / \mathrm{S}$ values as the sample without treatment dyed at $98^{\circ} \mathrm{C}$. These results 

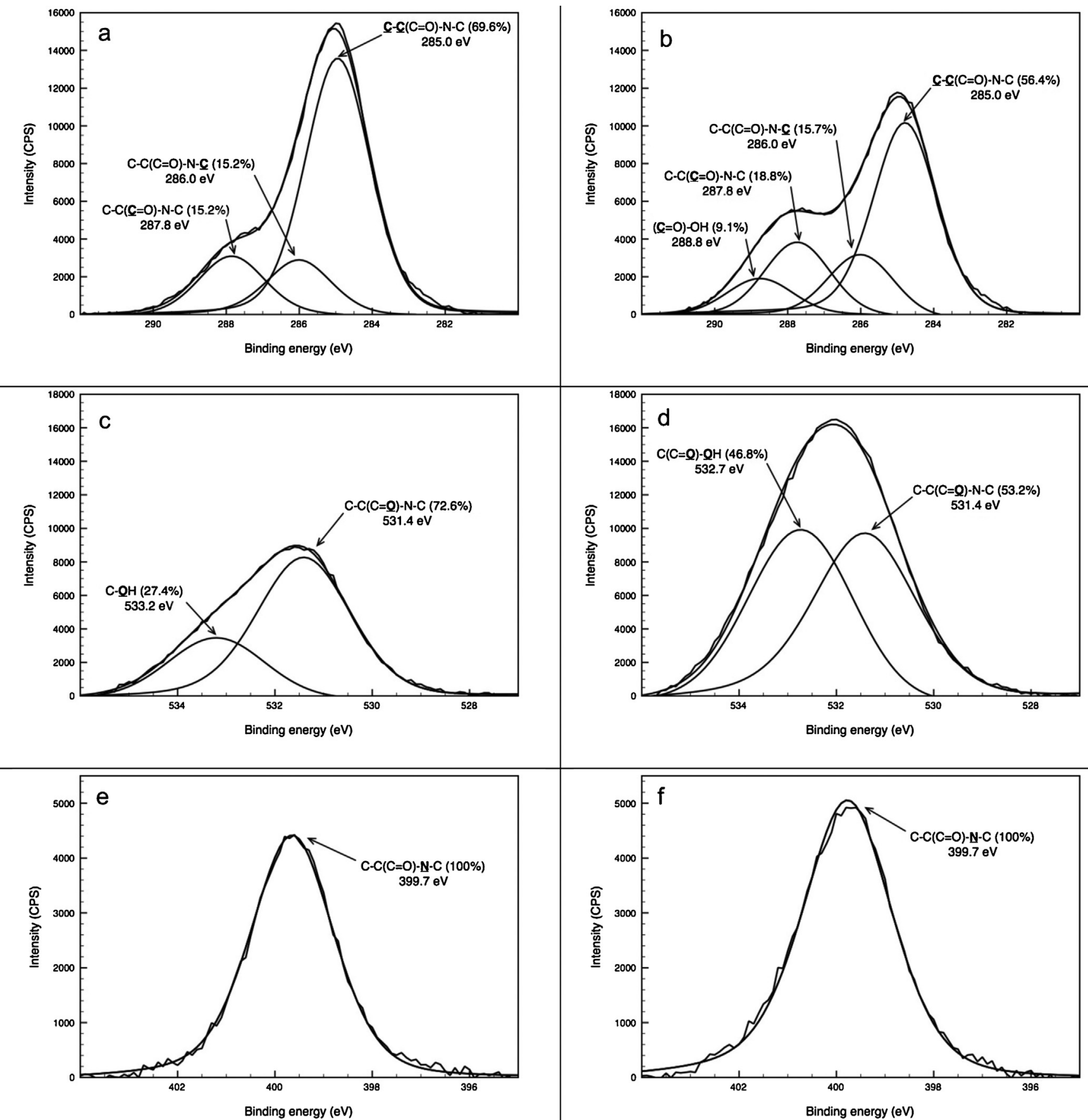

Fig. 3. High-resolution deconvoluted XPS spectra with relative areas of the C1s (a, b), O1s (c, d) and N1s (e, f) binding energy region of PA66 fibers surface before and after plasma treatment, respectively.

reveal that plasma treatment can improve polyamide saturation point.

Despite the fact that dye/water systems in dyeing processes are more complex than gas adsorption, in this case the Langmuir equation can be applied correlating the dye concentration in solution with the dye concentration on the fiber. Fig. 5 shows the effect of dye fiber uptake after $16 \mathrm{~h}$ in untreated and DBD plasma treated PA66 at different initial dye concentrations. Plasma treatment allows obtaining deeper colors at lower dye concentration and dyeing effluent. The dye uptake into the fibers demonstrates a graphical feature of an isothermal. Confirming that, the dyeing of polyamide fiber with Direct Orange 57 (high relative molecular weight and a high affinity to the fiber surface) is governed by the Langmuir isotherm. This allows the calculation of the affinity assuming that the dye is absorbed in a specific place of the fiber and cannot occupy the same position filled by another molecule. Plasma treatment causes a decrease in dye affinity for the PA66. These results are interesting from the point of view of the dye entrance in the fiber interior, because the affinity is inversely proportional to the diffusion coefficient. The dye/substrate bonding can occur due to specificity, in other words, due to the covalent, ionic, hydrogen or other weaker bonds. In many of these systems there may exist the overlap in more than one type of chemical bond. Thus, with the gradient increase, the diffusion rate should also be increasing. 


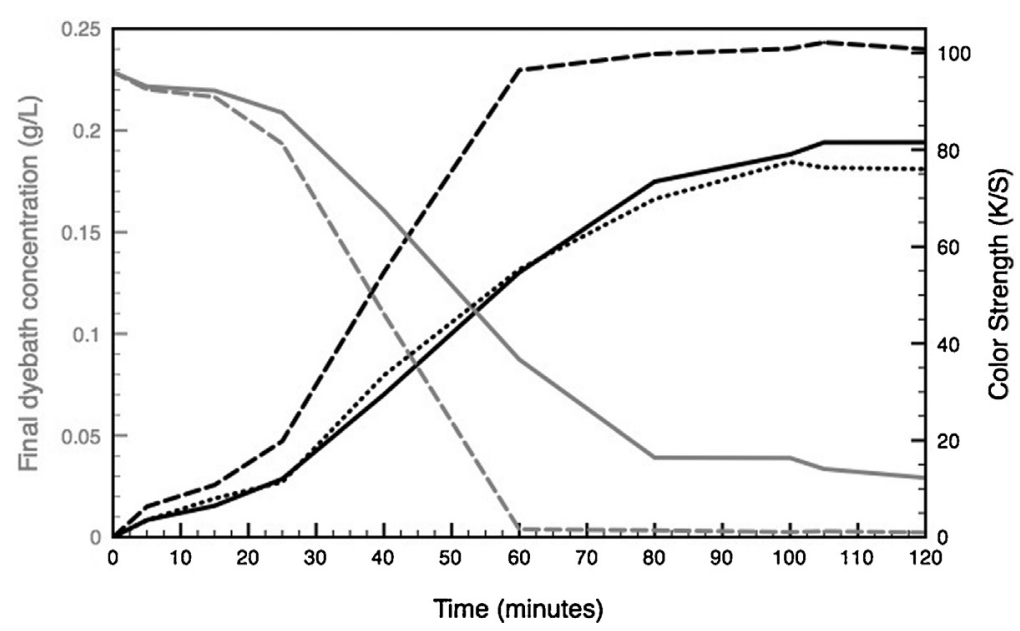

Fig. 4. Values of color strength (black lines) and dye exhaustion (gray lines) of untreated (solid lines) and plasma treated PA66 fabrics dyed at $98{ }^{\circ} \mathrm{C}$ (dashed lines) with $0.25 \mathrm{~g} \mathrm{~L}^{-1}$ of C.I. Direct Orange 57 in function of time and temperature. The dotted line represents the plasma treated PA66 fabrics dyed at $80{ }^{\circ} \mathrm{C}$.

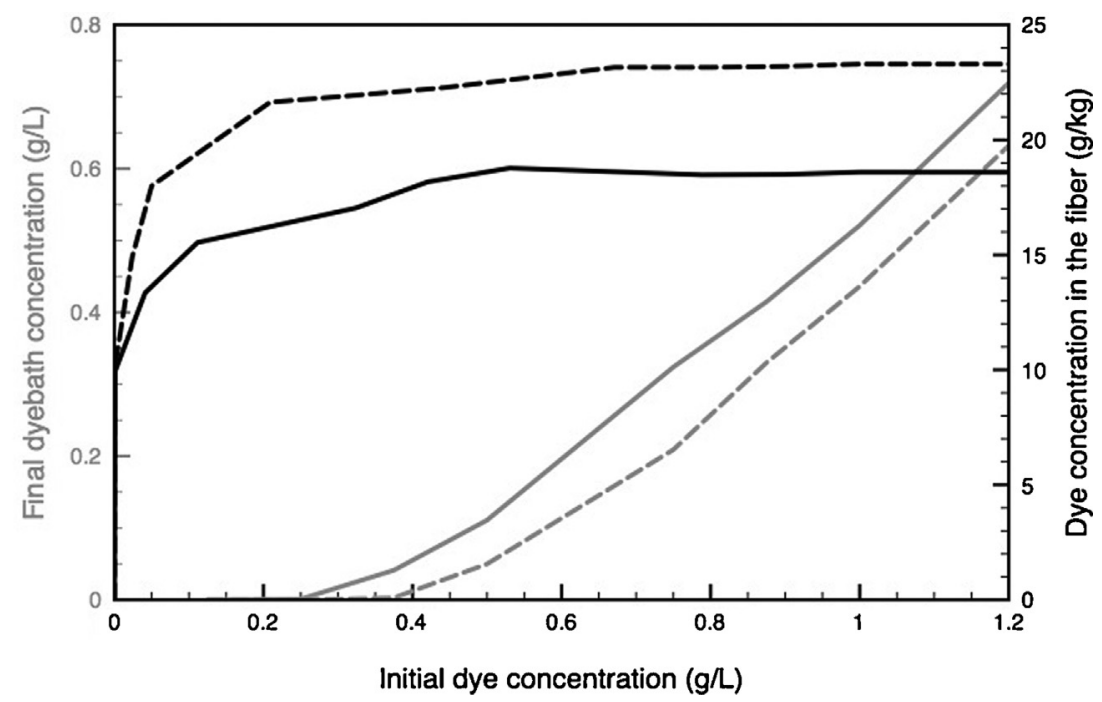

Fig. 5. Values of dye concentration in the fibers (black lines) and dye exhaustion (gray lines) of untreated (solid lines) and plasma treated (dashed lines) PA66 fabrics dyed for $16 \mathrm{~h}$ with C.I. Direct Orange 57 in function of different initial dye concentrations.

However, a decrease was observed in the diffusion coefficient because the dye molecules with high affinity have problems with being absorbed into the fibers due to strong medium retention. The decrease in affinity of Direct Orange 57 onto the PA66 fibers after plasma treatment could be one of the causes for the increased diffusion of the dye into the fiber. Moreover, the presence of plasma etched acidic species in solution may be also responsible for protonation of more $\mathrm{NH}_{2}$ groups, which increase the absorption and diffusion of direct dyes to the fiber.

The level of dye penetration into PA66 fibers during dye process was visualized by fluorescence microscopy. The cross-section images of the dyed plasma treated fibers in Fig. $6 \mathrm{~b}$ show widespread dye penetration into the interior of the PA66 fibers only after $40 \mathrm{~min}$. On the other hand, the untreated samples exhibit a ring effect with high surface concentration and low dye penetration. This ring of accumulated dye on the fiber surface causes a blocking effect that reduces dye penetration. This effect is reduced after plasma treatment due to the creation of both chemical modifications, resulting in breakage of the polyamide chains, and micro-channels that favor dye diffusion into the fiber interior. Efficient penetration of the dye molecules into textile fibers is essential to optimize color strength in wet and dry conditions and to reduce time and temperature of the dyeing process. Table 3 presents the results obtained by washing, light and rubbing fastness of the polyamide samples with and without plasma treatment. Despite the rapid dye diffusion, the results of washing fastness for direct dye Orange 57 in PA66 fabrics with three different concentrations are very good, confirming the high level of dye fixation. The results of wet and dry rubbing fastness in plasma-treated dyed fabrics are also very good. The value of 5 in the gray scale was obtained for all the samples. The light fastness of PA66 fabrics was evaluated from 2 to $48 \mathrm{~h}$. Even after short periods of light exposure $(2 \mathrm{~h})$, dye degradation was lower in the DBD treated samples. After $48 \mathrm{~h}$ of exposure DBD treated samples presented lower dye degradation, when compared with control samples especially at low and high concentrations $\left(0.25\right.$ and $\left.0.75 \mathrm{~g} \mathrm{~L}^{-1}\right)$. The surface modification of the PA66 fabric after DBD plasma treatment allows obtaining darker colors, with a better level of staining when comparing with the untreated sample.

\subsection{Dyeing mechanism}

PA66 is a semicrystalline thermoplastic condensation copolymers formed by reacting equal parts of two monomers (hexane-1, 
Table 3

Fastness properties of PA66 samples dyed with 0.25, 0.5 and $0.75 \mathrm{~g} \mathrm{~L}^{-1}$ of C.I. Direct Orange 57, with and without DBD treatment.

\begin{tabular}{lll}
\hline & Untreated $0.25,0.5,0.75 \mathrm{gL}^{-1}$ & Plasma treated $0.25,0.5,0.75 \mathrm{~g} \mathrm{~L}-1$ \\
\hline Rubbing fastness (gray scale) & $5,5,5$ & $5,5,5$ \\
Washing fastness - Color change (gray scale) & $5,4-5,4-5$ & $4-5,4-5,5$ \\
Washing fastness - Cotton staining (gray scale) & $4-5,4,3-4$ & $5,4,4-5$ \\
Light fastness $\left(\Delta E^{*}\right.$ after $2 \mathrm{~h}$ ) & $3.1,3.0,3.7$ & $1.1,1.4,2.4$ \\
Light fastness $\left(\Delta E^{*}\right.$ after $\left.12 \mathrm{~h}\right)$ & $5.8,4.8,6.6$ & $5.1,4.4,3.7$ \\
Light fastness $\left(\Delta E^{*}\right.$ after $\left.24 \mathrm{~h}\right)$ & $6.7,5.4,7.5$ & $5.3,4.9,4.6$ \\
Light fastness $\left(\Delta E^{*}\right.$ after $\left.48 \mathrm{~h}\right)$ & $7.5,5.8,8.2$ & $5.9,5.1,5.3$ \\
\hline
\end{tabular}

Temperature $\left({ }^{\circ} \mathrm{C}\right)$

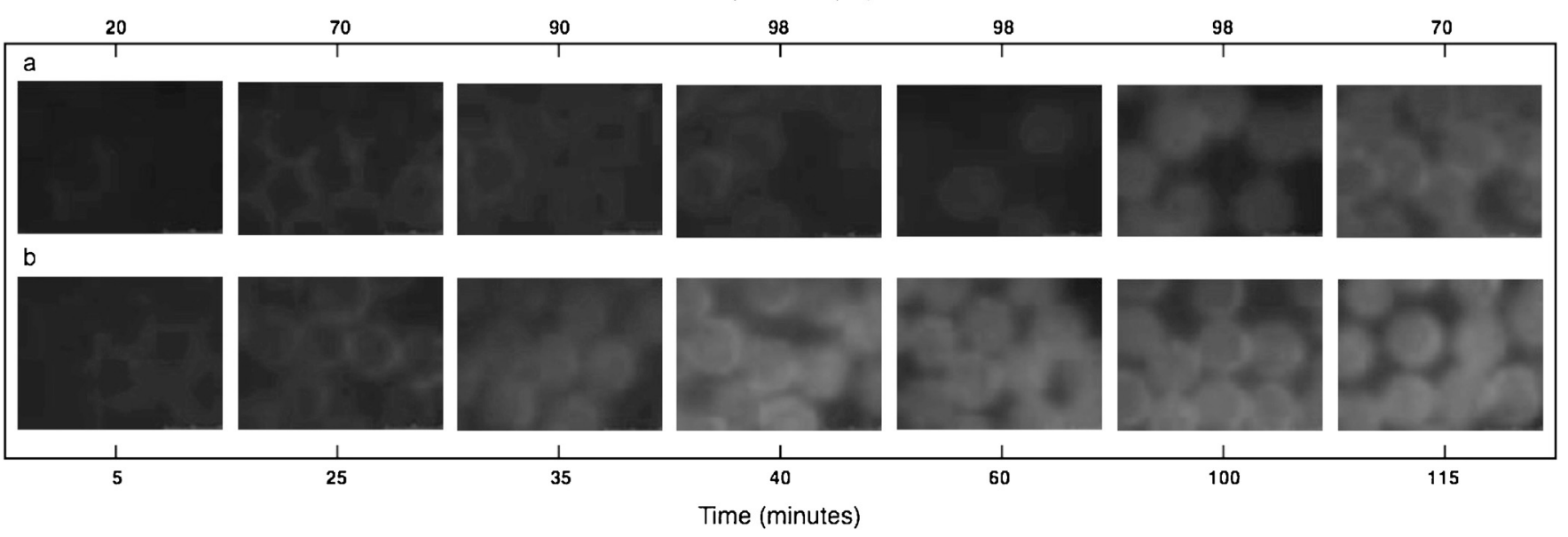

Fig. 6. Fluorescence microscopic images of the cross-section of untreated (a) and plasma treated (b) PA66 fibers taken during the dyeing process with C.I. Direct Orange 57 (original magnification $40 \times$ ).
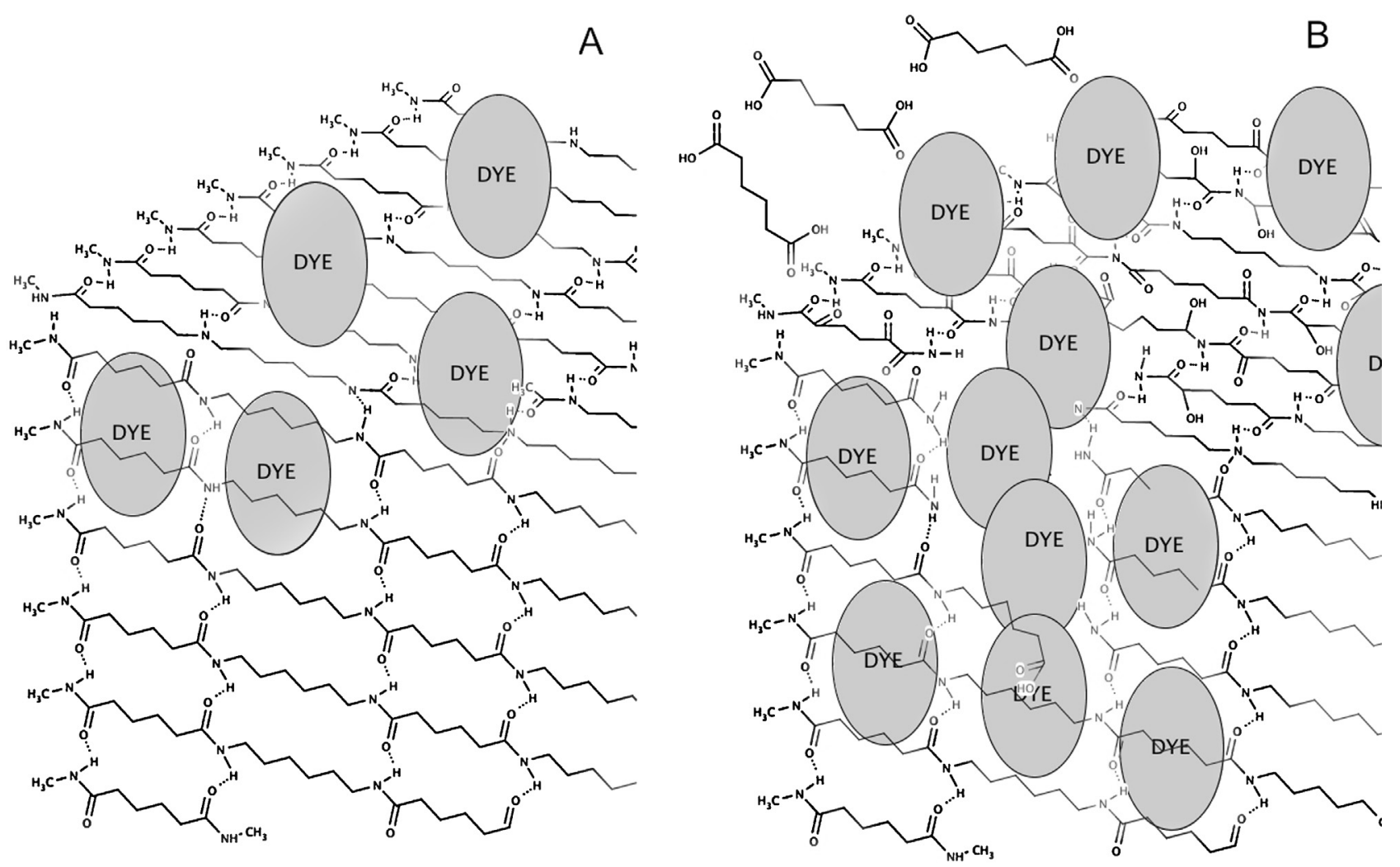

Fig. 7. Dyeing mechanism of nylon 66 fiber before (A) and after (B) plasma treatment. After plasma discharge, nylon 66 displays a significant amount of oxygen covalently bonded onto its surface and the presence of micro-channels that promote a deeper diffusion of the dye into the fibers. Acidic and partially soluble species are also released in the dyebath. 
6-diamine and hexanedioic acid), each of which containing 6 carbon atoms. However, only when drawn into fibers nylons become almost entirely crystalline. Its long molecular chain results of more sites for hydrogen bonds between the carbonyl oxygens and amide hydrogens, creating a chemical dense structure with small, evenly spaced pores. This is the reason why PA66 fibers are usually difficult to dye. On the other hand, once dyed nylon has superior color fastness and is less susceptible to fading from sunlight and ozone and to yellowing from nitrous oxide. The results of physico-chemical, morphological and dyeing properties indicate a possible dyeing mechanism of plasma treated PA66, which is shown schematically in Fig. 7. Due to the reduced surface barrier in plasma treated polyamide fibers, higher amount of dye is able to diffuse quickly through the top layer compared to the untreated polyamide showing an increase of around $25 \%$ in dye uptake. Since the bulk properties of the PA66 fabric do not change after plasma treatment, the increase of polar groups on the fibers surface seems to be responsible for enhancing direct dye diffusion onto and into the fibers. Moreover, the significant acidification of the media due to the plasma-etching generated species of plasma treated PA66 ( $\mathrm{pH}$ values decrease from 5.5 to 3.1) seems to promote the formation of additional $\mathrm{NH}_{3}{ }^{+}$groups on the polyamide surface and could also explain the greater dye affinity of these fibers. This hypothesis is confirmed by the $80 \%$ higher color yield in terms of $\mathrm{K} / \mathrm{S}$ values and by the fluorescence microscopic images of the cross-sections of the dyed plasma-treated PA66. However, other structural and chemical changes affected dye accessibility such as the creation of micro-channels, enhanced micro-roughness and reduction of glass transition temperature. The formation of these plasma-generated low-molecular weight organic molecules can be also considered as a sort of dye "carriers", aiding the diffusion of the dye into the fibers through the plasma generated micro-channels and open paths. The XPS analysis confirms that the PA66 chain scission in $\mathrm{C}-\mathrm{N}$ also promotes the formation of reactive species increasing the concentration of polar groups mainly by the incorporation of oxygen atoms near the surface of the fabric. In addition, as confirmed by FTIR analysis, the acidic character of these small molecules can be a direct influence in the creation of more amino groups available inside of the molecular structure of PA66. Therefore, when a channel is created in the PA66 chain, the diffusing species are allowed to react with the new bonds in the broken chains as well as with the exposed PA66 chain below [57].

\section{Conclusions}

This research shows that a relatively low DBD plasma dosage, of around $2.5 \mathrm{~kW} \mathrm{~min} \mathrm{~m}^{-2}$, can generate significant physico-chemical changes onto PA66 fiber surfaces increasing their wettability, surface energy and adhesion. SEM images show that the topography of the PA66 fibers was uniformly altered after plasma treatment increasing surface roughness. The introduction of polar groups, mainly oxygen from atmospheric air, is responsible for the improved wettability of the plasma modified PA66. This is clearly demonstrated by the significant increase in the surface-energy polar component and by the increase in intensity and broadening of the $\mathrm{C}=\mathrm{O}$ stretching band as well as of the bending band of the $\mathrm{O}=\mathrm{C}-\mathrm{N}$ groups in the samples after blind dyeing. On the other hand, the increased intensities of the $\mathrm{N}-\mathrm{H}$ and asymmetric and symmetric $\mathrm{C}-\mathrm{H}$ stretching vibration bands as well as the $\mathrm{pH}$ decrease in the aqueous extraction demonstrate the release of acidic low-molecular weight etched species. The deconvolution analysis of $\mathrm{C} 1 \mathrm{~s}, \mathrm{O} 1 \mathrm{~s}$ and N1s XPS peaks of the plasma treated PA66 confirms the decrease in the aliphatic functional groups and the remarkable increase of the oxygen bonded to carbon atoms. FTIR and XPS results seem to indicate that the preferred chain scission site is $\mathrm{C}-\mathrm{N}$ bond. The physico-chemical, morphological and dyeing results lead to a new understanding of the dyeing mechanism of the plasma treated polyamide 6,6 . The DBD breakages of the polyamide chains generate micro-channels and open paths that favor the dye diffusion into the fiber interior. At the same time, plasma generated low-molecular weigh aliphatic chains tend, by oxidation, to form acidic and partially soluble species that act as a sort of dye "carrier" into the fiber. As observed in the fluorescence microscopy crosssection images, the application of direct dye in DBD treated PA66 promotes a widespread improvement of dye exhaustion and dye diffusion deep into the interior of the fibers. The kinetics of dyeing is quicker but leveled with high rubbing and washing fastness quality. This is a great advantage for all anionic dyes since darker shades are obtainable using lower amounts of dyestuffs at lower temperature and dyeing time.

\section{Acknowledgements}

Fernando Oliveira (SFRH/BD/65254/2009) acknowledges Fundação para a Ciência e Tecnologia, Portugal, for its doctoral grant financial support. Andrea Zille (C2011-UMINHO-2C2T-01) acknowledges funding from Programa Compromisso para a Ciência 2008, Portugal.

\section{References}

[1] M. Gorjanc, M. Gorensek, P. Jovancic, M. Mozetic, Multifunctional textiles modification by plasma, dyeing and nanoparticles, in: M. Günay (Ed.), EcoFriendly Textile Dyeing and Finishing, InTech, 2013.

[2] F. Denes, Macromolecular plasma-chemistry: an emerging field of polymer science, Prog. Polym. Sci. 29 (2004) 815-885.

[3] G. Borcia, N. Dumitrascu, G. Popa, Influence of helium-dielectric barrier discharge treatments on the adhesion properties of polyamide-6 surfaces, Surf. Coat. Technol. 197 (2005) 316-321.

[4] R. Morent, N. De Geyter, J. Verschuren, K. De Clerck, P. Kiekens, C. Leys, Non-thermal plasma treatment of textiles, Surf. Coat. Technol. 202 (2008) 3427-3449.

[5] C.X. Jia, P. Chen, W. Liu, B. Li, Q.A. Wang, Surface treatment of aramid fiber by air dielectric barrier discharge plasma at atmospheric pressure, Appl. Surf. Sci. 257 (2011) 4165-4170.

[6] G. Borcia, C.A. Anderson, N.M.D. Brown, Surface treatment of natural and synthetic textiles using a dielectric barrier discharge, Surf. Coat. Technol. 201 (2006) 3074-3081.

[7] N.Y. Cui, D.J. Upadhyay, C.A. Anderson, N.M.D. Brown, Study of the surface modification of a nylon-6,6 film processed in an atmospheric pressure air dielectric barrier discharge, Surf. Coat. Technol. 192 (2005) 94-100.

[8] U. Kogelschatz, Dielectric-barrier discharges: their history, discharge physics, and industrial applications, Plasma Chem. Plasma Process. 23 (2003) 1-46.

[9] K. Schneider, C. Hafner, I. Jäger, Mutagenicity of textile dye products, J. Appl. Toxicol. 24 (2004) 83-91.

[10] H.A. Karahan, E. Ozdogan, A. Demir, H. Ayhan, N. Seventekin, Effects of atmospheric plasma treatment on the dyeability of cotton fabrics by acid dyes, Color Technol. 124 (2008) 106-110.

[11] D. Sun, G.K. Stylios, Effect of low temperature plasma treatment on the scouring and dyeing of natural fabrics, Text. Res. J. 74 (2004) 751-756.

[12] S. Nourbakhsh, P. Valipour, M.E. Yazdanshenas, A.G. Ebadi, Dyeability improvement of cationized corona discharge treated cotton fabric, Asian J. Chem. 20 (2008) 3543-3548.

[13] N.A. Ibrahim, M.M. Hashem, M.A. Eid, R. Refai, M. El-Hossamy, B.M. Eid, Ecofriendly plasma treatment of linen-containing fabrics, J. Text. I 101 (2010) 1035-1049.

[14] J. Yip, K. Chan, K.M. Sin, K.S. Lau, Study of physico-chemical surface treatments on dyeing properties of polyamides. Part 1: Effect of tetrafluoromethane low temperature plasma, Color Technol. 118 (2002) 26-30.

[15] F.R. Oliveira, A.P. Souto, N. Carneiro, J.H.O. Nascimento, Surface modification on polyamide 6.6 with double barrier discharge (DBD) plasma to optimise dyeing process by direct dyes, Mater. Sci. Forum 636-637 (2010) 846-852.

[16] A.P. Souto, F. Ribeiro, N. Carneiro, Polyamide 6.6 modified by DBD plasma treatment for anionic dyeing processes, in: P.J. Hauser (Ed.), Textile Dyeing, InTech, 2011, pp. 241-260.

[17] A. Raffaele-Addamo, E. Selli, R. Barni, C. Riccardi, F. Orsini, G. Poletti, L. Meda, M.R. Massafra, B. Marcandalli, Cold plasma-induced modification of the dyeing properties of poly(ethylene terephthalate) fibers, Appl. Surf. Sci. 252 (2006) 2265-2275.

[18] M.M. Hossain, J. Mussig, A.S. Herrmann, D. Hegemann, Ammonia/acetylene plasma deposition: an alternative approach to the dyeing of poly(ethylene terephthalate) fabrics at low temperatures, J. Appl. Polym. Sci. 111 (2009) 2545-2552. 
[19] S. Shahidi, M. Ghoranneviss, B. Moazzenchi, A. Rashidi, D. Dorranian, Effect of using cold plasma on dyeing properties of polypropylene fabrics, Fiber Polym. 8 (2007) 123-129.

[20] N. Yaman, E. Ozdogan, N. Seventekin, H. Ayhan, Plasma treatment of polypropylene fabric for improved dyeability with soluble textile dyestuff, Appl. Surf. Sci. 255 (2009) 6764-6770.

[21] Y. Iriyama, T. Mochizuki, M. Watanabe, M. Utada, Plasma treatment of silk fabrics for better dyeability, J. Photopolym. Sci. Technol. 15 (2002) 299-306.

[22] Z.S. Cai, Y.P. Qiu, Dyeing properties of wool fabrics treated with atmospheric pressure plasmas, J. Appl. Polym. Sci. 109 (2008) 1257-1261.

[23] M.M. El-Zawahry, N.A. Ibrahim, M.A. Eid, The impact of nitrogen plasma treatment upon the physical-chemical and dyeing properties of wool fabric, Polym. Plast. Technol. 45 (2006) 1123-1132.

[24] D. Jocic, S. Vilchez, T. Topalovic, R. Molina, A. Navarro, P. Jovancic, M.R. Julia, P. Erra, Effect of low-temperature plasma and chitosan treatment on wool dyeing with acid red 27, J. Appl. Polym. Sci. 97 (2005) 2204-2214.

[25] S. Ratnapandian, L.J. Wang, S.M. Fergusson, M. Naebe, Effect of atmospheric plasma treatment on pad-dyeing of natural dyes on wool, Text. Bioeng. Inf. Symp. Proc. 1-3 (2011) 780-786.

[26] M. Radetic, P. Jovancic, N. Puac, Z.L. Petrovic, Environmental impact of plasma application to textiles, J. Phys. Conf. Ser. 71 (2007) U214-U224.

[27] L. Razafimahefa, I. Vromana, P. Viallier, Mechanisms of fixation of dyestuffs in polyamide 6.6 fibres, Color Technol. 119 (2003) 10-13.

[28] M. Xi, Y.L. Li, S.Y. Shang, D.H. Li, Y.X. Yin, X.Y. Dai, Surface modification of aramid fiber by air DBD plasma at atmospheric pressure with continuous online processing, Surf. Coat. Technol. 202 (2008) 6029-6033.

[29] D. Pappas, A. Bujanda, J.D. Demaree, J.K. Hirvonen, W. Kosik, R. Jensen, S. McKnight, Surface modification of polyamide fibers and films using atmospheric plasmas, Surf. Coat. Technol. 201 (2006) 4384-4388.

[30] M.B.O. Riekerink, J.G.A. Terlingen, G.H.M. Engbers, J. Feijen, Selective etching of semicrystalline polymers: CF4 gas plasma treatment of poly(ethylene), Langmuir 15 (1999) 4847-4856.

[31] Y.J. Hwang, S. Matthews, M. McCord, M. Bourham, Surface modification of organic polymer films treated in atmospheric plasmas, J. Electrochem. Soc. 151 (2004) C495-C501.

[32] S.A. Smirnov, V.V. Rybkin, I.V. Kholodkov, Simulation of the processes of formation and dissociation of neutral particles in air plasma: vibrational kinetics of ground states of molecules, High Temp. 40 (2002) 161-165.

[33] X.M. Li, T. He, M. Crego-Calama, D.N. Reinhoudt, Conversion of a metastable superhydrophobic surface to an ultraphobic surface, Langmuir 24 (2008) 8008-8012.

[34] F.M. Fowkes, Citation classic - attractive forces at interfaces, Curr. Contents/Phys. Chem. Earth Sci. (1980) 12.

[35] W. Qufu, W. Yingying, Y. Qin, Y. Liangyan, Functionalization of textile materials by plasma enhanced modification, J. Ind. Text. 36 (2007) 301-309.

[36] D.J. Upadhyay, N.Y. Cui, C.A. Anderson, N.M.D. Brown, A comparative study of the surface activation of polyamides using an air dielectric barrier discharge, Colloids Surf. A 248 (2004) 47-56.

[37] K.H. Kale, A.N. Desai, Atmospheric pressure plasma treatment of textiles using non-polymerising gases, Indian J. Fibre Text. 36 (2011) 289-299.

[38] J. Charles, G.R. Ramkumaar, S. Azhagiri, S. Gunasekaran, FTIR and thermal studies on nylon-66 and 30\% glass fibre reinforced nylon-66, E-J. Chem. 6 (2009) 23-33.

[39] K.H. Kale, S.S. Palaskar, Structural studies of plasma polymers obtained in pulsed dielectric barrier discharge of TEOS and HMDSO on nylon 66 fabrics J. Text. I 103 (2012) 1088-1098.
[40] M.C. Gupta, R.R. Pandey, Gamma-irradiation of nylon-6, J. Polym. Sci. Polym. Chem. 26 (1988) 491-502.

[41] Y. Zheng, H.Y. Liu, P.V. Gurgel, R.G. Carbonell, Polypropylene nonwoven fabrics with conformal grafting of poly(glycidyl methacrylate) for bioseparations, J. Membr. Sci. 364 (2010) 362-371.

[42] V. Goodarzi, S. Hassan Jafari, H. Ali Khonakdar, B. Ghalei, M. Mortazavi, Assessment of role of morphology in gas permselectivity of membranes based on polypropylene/ethyl vinyl acetate/clay nanocomposite, J. Membr. Sci. 445 (2013) 76-87.

[43] E.D. Mattos, I. Vigano, R.D.L. Dutra, M.F. Diniz, K. Iha, Application of FTIR methodologies of transmission and photoacoustic to the characterization of highly energetic materials - Part II, Quim. Nova 25 (2002) 722-728.

[44] L. Zhu, C. Wang, Y. Qiu, Influence of the amount of absorbed moisture in nylon fibers on atmospheric pressure plasma processing, Surf. Coat. Technol. 201 (2007) 7453-7461.

[45] Q. Zhou, K. Wang, L.S. Loo, Investigation of surface properties of plasmamodified polyamide 6 and polyamide 6/layered silicate nanocomposites, J. Mater. Sci. 46 (2010) 3084-3093.

[46] G. Borcia, N. Dumitrascu, G. Popa, Influence of dielectric barrier discharge treatments on the surface properties of polyamide-6 films, J. Optoelectron. Adv. Mater. 7 (2005) 2535-2538.

[47] U. Lommatzsch, D. Pasedag, A. Baalmann, G. Ellinghorst, H.E. Wagner, Atmospheric pressure plasma jet treatment of polyethylene surfaces for adhesion improvement, Plasma Process. Polym. 4 (2007) S1041-S1045.

[48] P.K. Panda, D. Rastogi, M. Jassal, A.K. Agrawal, Effect of atmospheric pressure helium plasma on felting and low temperature dyeing of wool, J. Appl. Polym. Sci. 124 (2012) 4289-4297.

[49] M.I. Mejia, J.M. Marin, G. Restrepo, C. Pulgarin, E. Mielczarski, J. Mielczarski, I. Stolitchnov, J. Kiwi, Innovative UVC light (185 nm) and radio-frequency-plasma pretreatment of nylon surfaces at atmospheric pressure and their implications in photocatalytic processes, ACS Appl. Mater. Interfaces 1 (2009) 2190-2198.

[50] R. Morent, N. De Geyter, C. Leys, L. Gengembre, E. Payen, Surface modification of non-woven textiles using a dielectric barrier discharge operating in air, helium and argon at medium pressure, Text. Res. J. 77 (2007) 471-488.

[51] S.B. Idage, M.D. Dumbre, R. Singh, Photo-oxidative degradation of nylon 6.6 under accelerated weathering study by X-ray photoelectron spectroscopy, in Proceedings of Recent Advances in Polymers and Composites, Allied Publishers, 2000, p. 703.

[52] F.R. Oliveira, L. Erkens, R. Fangueiro, A.P. Souto, Surface modification of banana fibers by DBD plasma treatment, Plasma Chem. Plasma Process. 32 (2012) 259-273.

[53] N.Y. Cui, N.M.D. Brown, Modification of the surface properties of a polypropylene (PP) film using an air dielectric barrier discharge plasma, Appl. Surf. Sci. 189 (2002) 31-38.

[54] J.D. Liao, C.Y. Chen, Y.T. Wu, C.C. Weng, Hydrophilic treatment of the dyed nylon-6 fabric using high-density and extensible antenna-coupling microwave plasma system, Plasma Chem. Plasma Process. 25 (2005) 255-273.

[55] A. Tarasova, P. Hamilton-Brown, T. Gengenbach, H.J. Griesser, L. Meagher Colloid probe AFM and XPS study of time-dependent aging of amine plasma polymer coatings in aqueous media, Plasma Process. Polym. 5 (2008) 175-185.

[56] D. Sun, G.K. Stylios, Investigating the plasma modification of natural fiber fabrics-the effect on fabric surface and mechanical properties, Text. Res. J. 75 (2005) 639-644.

[57] R. Dorai, M.J. Kushner, A model for plasma modification of polypropylene using atmospheric pressure discharges, J. Phys. D: Appl. Phys. 36 (2003) 666-685. 